Progress in Flight Physics 5 (2013) 493-516

DOI: $10.1051 /$ eucass/201305493

(C) Owned by the authors, published by EDP Sciences, 2013

\title{
NONEQUILIBRIUM COMPUTATIONAL FLOWFIELD ANALYSIS FOR THE DESIGN OF MARS MANNED ENTRY VEHICLES
}

\author{
A. Viviani ${ }^{1}$ and G. Pezzella ${ }^{2}$ \\ ${ }^{1}$ Seconda Università di Napoli (SUN) \\ 29 via Roma, Aversa 81031, Italy \\ ${ }^{2}$ Centro Italiano Ricerche Aerospaziali \\ via Maiorise, Capua 81043, Italy
}

\begin{abstract}
This paper deals with the aerodynamic exploration analysis of innovative unusual vehicle concepts, suitable for a manned Mars entry, aiming to support design studies of planetary entry systems. In this framework, two lifting body (LB) configurations have been analyzed so far. Several fully three-dimensional (3D) computational fluid dynamics (CFD) analyses, both for a perfect gas model and for a nonequilibrium reacting gas mixture model, have been performed. The flowfield environment past the exploration vehicle for an assigned Mars entry scenario has been addressed. To this end, a wide range of flow conditions, including different angles of attack (AoA), various Mach numbers, and Reynolds numbers, have been investigated. Results reported in the paper are useful for providing numerical data for further understanding the fluid dynamics processes, as those of high enthalpy flow and vehicle shape effects, involved in the design analysis of LB vehicles for the exploration of Mars.
\end{abstract}

\section{INTRODUCTION}

This paper reports about the results of aerodynamic design analyses of two manned braking systems (MBS) entering the Mars atmosphere, with the aim of supporting design studies of a planetary entry system. Two unusual LB configurations with a rounded edge delta-like cross section have been analyzed. The preliminary aerodynamic analyses have been focused on flight conditions compatible with a manned mission entering the Mars atmosphere. However, neither the mission architecture needed to reach Mars from Earth or the Earth's neighboring space, nor the surface exploration have been addressed. 
All the design analyses have been performed at several levels. Indeed, vehicle aerodynamic assessment has been extensively addressed through simplified design approach as, e.g., hypersonic panel methods (HPM); then, a number of fully 3D CFD simulations, both with Euler and Navier-Stokes approximations, of the hypersonic flowfield past the entry vehicle have been performed.

The results herein provided have been obtained for a Mars entry scenario compliant with an approach to the red planet both by a direct planetary entry and an entry after aerobraking [1]. These results may be used to provide numerical data for understanding the requirements for the human exploration of Mars [2].

\section{VEHICLE CONFIGURATION SELECTION}

The vehicle concepts investigated in this work are atypical LB configurations (Fig. 1). Figure $1 a$ shows the vehicle named LB-A while Fig. $1 b$ shows the vehicle named LB-B.

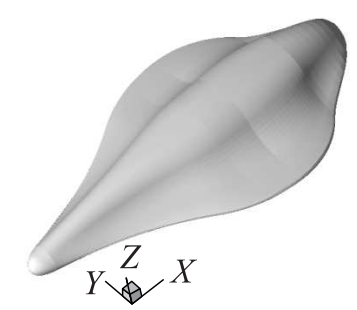

(a)
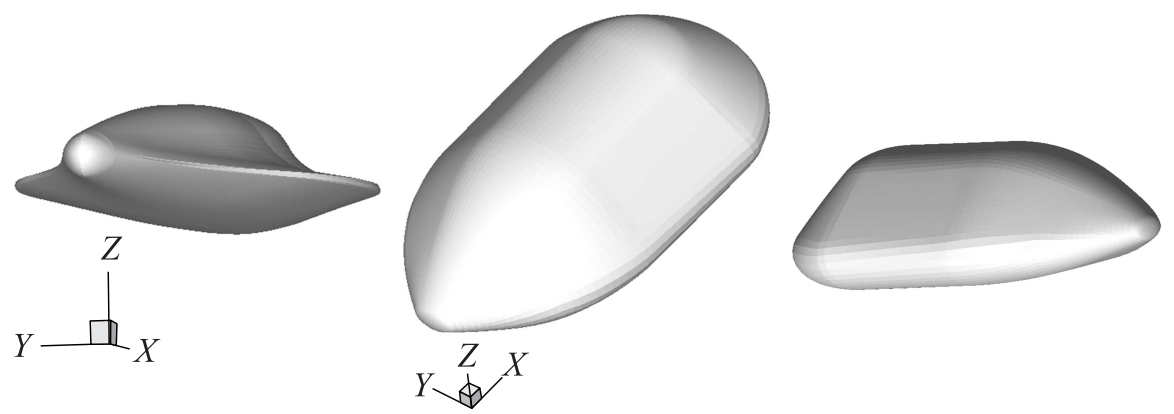

(b)

Figure 1 Vehicle configurations LB-A $(a)$ and LB-B $(b)$ 


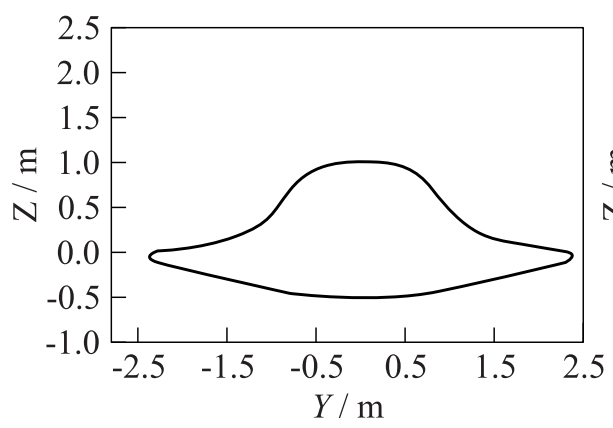

(a)

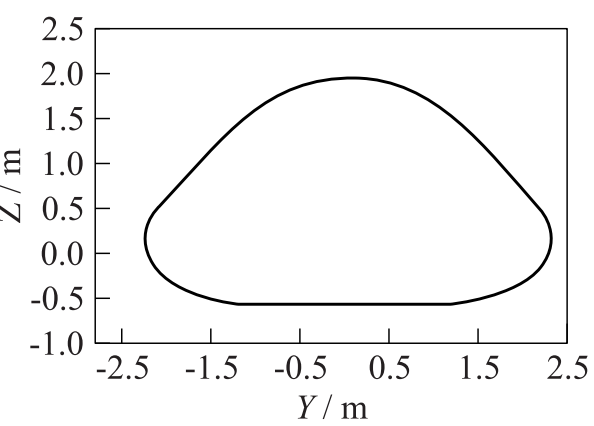

(b)

Figure 2 Vehicle cross sections [3] at $x=5(a)$ and $4.74 \mathrm{~m}(b)$

They represent the best configuration obtained by optimization processes performed at Dipartimento di Ingegneria Aerospaziale e Meccanica of Second University of Naples in the framework of a design study of planetary entry systems [3]. The main objective of this study is the searching for the minimum weight configuration taking into account both the vehicle thermal and operational constraints, as discussed in [3]. Note that minimizing the vehicle weight is an important figure of merit within the optimization processes for the design of a Mars exploration mission.

Each vehicle features an aerodynamic configuration with a compact body with a rounded edge delta-like cross section as shown in Fig. 2 [3].

The proposed concepts represent a rather conventional configuration characterized by a low nose camber and a flat bottomed surface in order to increase the overall vehicle hypersonic performance. The forebody is characterized by simple cone-sphere geometry with smooth streamlined surfaces on the upper and lower side of the vehicle (Fig. $3 a$ ). A very preliminary internal layout for a crew of three/four astronauts is also reported in Fig. $3 b$ (for the LB-B configuration only).

The nose radius of both vehicle concepts reads $0.3 \mathrm{~m}$.

Each vehicle is thought out for a small crew entering into the Mars atmosphere from a low orbit and performing a variable AoA entry trajectory.

For instance, during the entry maneuver, the vehicle experiences a highspeed and high-temperature flow. As a consequence, three main design topics have been focused for the safety and the performance: the structural integrity of the vehicle; livable conditions for the crew; and the minimization of the vehicle structural weight. Considering that the peaks of both structural and thermal stresses are generally reached during the hypersonic flight and the low speed flight is assumed being performed by the use of a parachutes system, only the flight hypersonic regime has been analyzed. 


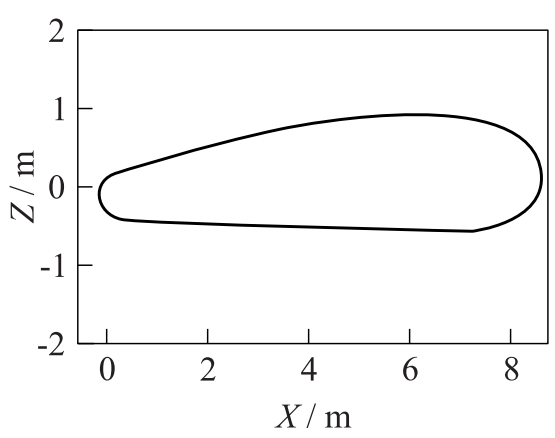

(a)

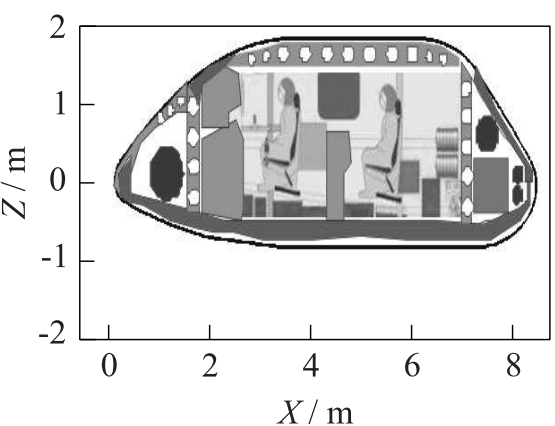

(b)

Figure 3 Vehicles centerline with internal layout for the LB-B configuration only

The pursuit of a feasible and livable minimum weight configuration has preliminarily required the definition of a well-suited parametric model for both the vehicle aerodynamic shape and for thickness distribution of the thermal insulating material.

The structural stresses are considered by the mean of the asymptotic dynamic pressure and the normal load factors peaks, and crew liveability conditions are accounted for by controlling that the inner temperature remains locally below a duly imposed value. An optimization procedure, using Genetic Algorithm (GA), is performed to find the optimal configuration [3]. The vehicle shape is modeled by a suitable parametric model, based on Coons surfaces, and a parametric insulating material distribution is foreseen.

The entry trajectory is calculated by the three degree-of-freedom (3 dof) model for planetary unpowered gliding entry with no bank angle (see Fig. 5). The shape aerodynamics is performed via HPM and the thermal state of the external surface is determined by means of one-dimensional (1D) boundary layer model together with the hypothesis of radiative cooling at the vehicle wall. Some other tools able to evaluate the LB entry trajectory and the thermal analysis of the protection shield system have been also developed. The initial trajectory angle is considered as an optimization parameter. Furthermore, a parametric control of the AoA has been implemented.

A two-people crew has been considered for the LB-A concept. The crew layout into the reentry vehicle is not a priori fixed, but parameterized and managed by a discrete variable. The internal volume hosting the crew is set by the layout parameter while the volumetric efficiency is considered in the vehicle configuration selection, since, in the first order, the thermal protection system (TPS) and the structural weight are a direct function of the vehicle wetted area.

Then, the vehicle outer shape is defined in detail by about thirty parameters that perform an affine transformation of a spherical domain into a suitable 
aerodynamic shape [3]. The latter vehicle feature is an important design topic considering that, in the case of Mars entry, the basic problem is one of decelerating the vehicle sufficiently to allow for the tenuous nature of the atmosphere. As a result, the most significant vehicle requirement is a rather low ballistic coefficient, $\beta_{f}$.

As a consequence, the vehicle configurations must have very blunt shapes, as in the case of LB-B configuration, in order to maximize the vehicle aerodynamic drag, $C_{D}$.

Moreover, the vehicle must be also able to provide rather high aerodynamic lift to increase the allowable $\beta_{f}$ and, therefore, the payload weight, as in the case of the more streamlined configuration, LB-A [4].

Finally, for the Mars entry, heating is a determining factor in the vehicle configuration. Since manned vehicles are necessarily large, radiant heating becomes dominant for such entries if simply blunt vehicles are used. To avoid such heating, configurations such as those reported in Fig. 1 have been proposed considering that the radiative heat flux is linearly dependent on the geometrical radius of the vehicle nose [5].

\section{METHODOLOGY ANALYSIS AND USED TOOLS}

This work summarizes a contribution to the planetary exploration concept design dealing with the definition of both the preliminary aerodynamic (AEDB) and aerothermodynamic (ATDB) databases for a manned vehicle entering the Mars atmosphere, compliant with a phase-A design level [6]. The former (AEDB) is needed for the Flight Mechanics analyses, since it must be verified that the concept aerodynamic performance ensures that the vehicle stays within the load constraints (i. e., pressure, inertial and thermal loads) during the descent. The latter (ATDB) is needed for the TPS design analyses; in fact, as a vehicle flies through the descent to the red planet, its internal energy (i. e., potential plus kinetic) is converted into the thermal energy within the boundary layer close to the vehicle surface, so that a thermal-shield must be provided to the MBS in order to sustain this heat loading.

In this framework, different design approaches have been adopted. The aerodynamic performance of each concept has been assessed according to the SpaceBased design approach [7]. This design approach dictates the generation of a complete data set as function of a number of independent parameters such as: Mach number $\left(\mathrm{M}_{\infty}\right)$, Reynolds number $\left(\operatorname{Re}_{\infty}\right)$, AoA $(\alpha)$, and sideslip angle $(\beta)$.

On the other hand, the surface heat flux distributions for each MBS concept have been provided according to the Trajectory-Based design approach [7]. It dictates the generation of a complete ATDB by performing the aerothermal 
computations at a finite number of "critical" points selected along with the vehicle design trajectory.

An accurate aerodynamic and aerothermodynamic analysis for several flight conditions, however, is very complex and time consuming, and is not compatible with a Phase-A design study, in which fast predicting methods are mandatory. Therefore, aerodynamic and aerothermodynamic analyses have been mainly performed by means of engineering-based approach, by using a 3D panel methods code developed by CIRA [8]. Increasing the order of complexity, a number of detailed CFD simulations has been carried out to more deeply characterize the hypersonic aerothermal environment of the vehicles and to focus on some critical design aspects not predictable with simplified tools. Indeed, 3D Euler and Navier-Stokes numerical flowfield computations have been performed at different Mach numbers and AoA at the most critical flight conditions occurring during the descent trajectory.

\subsection{Engineering-Based Design Analysis}

Engineering based aerodynamic analysis has been extensively performed by using a 3D Panel Methods code, namely, CIRA SIM, based on the simplified Newtonian approach and local surface inclination methods (SIM). This tool, at high supersonic and hypersonic speeds, is able to accomplish the aerodynamic and aerothermodynamic analyses of a complex reentry vehicle configuration by using simplified approaches as local SIM and approximate boundary-layer methods, thus avoiding complex grid generation and CFD high time consumption [8]. Typical surface meshes of the MBS used for the engineering level computations are shown in Fig. 4. As shown, the vehicle surface is approximated by a system of planar and leading edge panels; the lowest level of geometry used in the analysis is a quadrilateral element.

The pressure acting on each panel of impact and shadow regions is evaluated by the user-specified compression-expansion method. Several methods, typical of hypersonics, are available such as those derived from Newtonian, Modified Newtonian, and Prandtl-Mayer theories [5]. In order to predict viscous contribution to aerodynamic forces and moments, the shear force is determined on each vehicle panel on the assumption of a laminar or turbulent flat plate [9].

In particular, the viscous calculation is performed along with streamlines, and the results are then interpolated at the panel centroids. The streamlines are generated starting from the inviscid surface velocities generated previously in the aerodynamic analysis phase.

Finally, the panel aeroheating is evaluated by means of approximate 1D Boundary-Layer Methods (BLM) as reference temperature and reference enthalpy ones, which are available both for laminar and turbulent boundary layer 


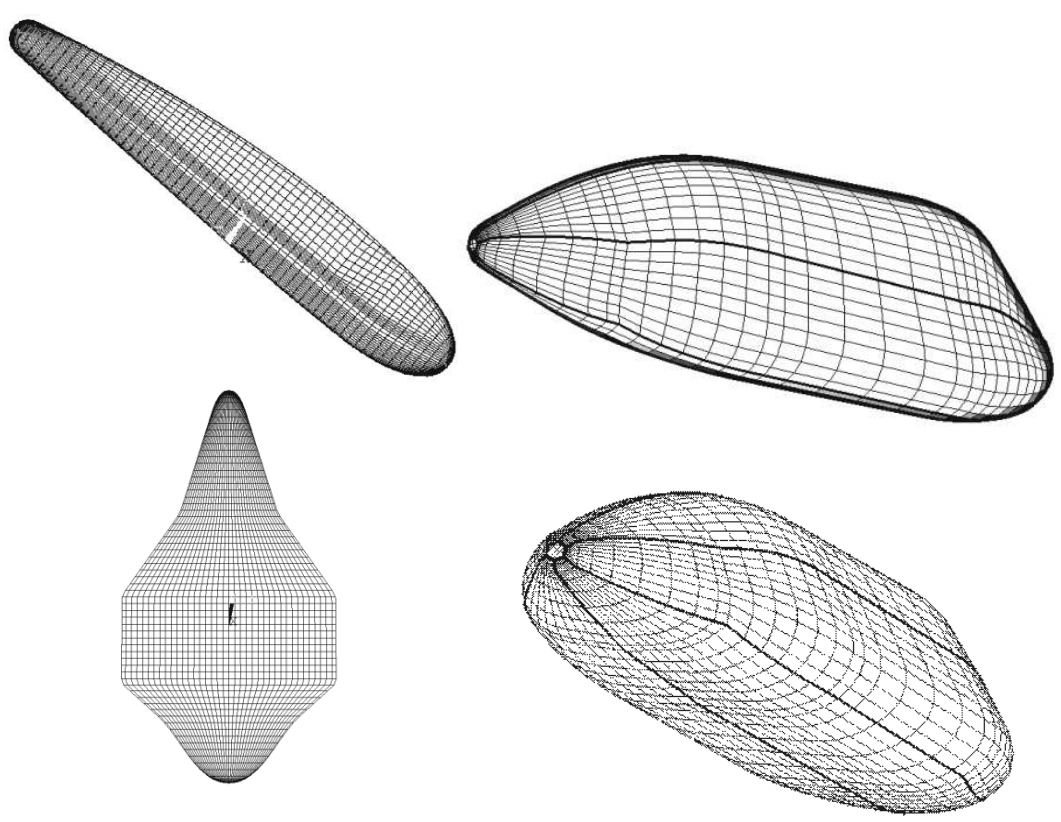

Figure 4 Examples of surface mesh used for engineering-based design analyses

assumptions. Indeed, the generic vehicle component may be modeled as either a flat plate or a leading edge by selecting the appropriate BLM. The Mangler factor is used to transform the solution of two-dimensional (2D) boundary layers to the axially symmetrical case [9].

\subsection{Design Analysis based on Computational Fluid Dynamics}

The CFD design analyses of vehicle concepts have been carried out by using the commercial code FLUENT [10]. A number of user defined functions (UDFs), developed in order to perform numerical computations with a thermochemical nonequilibrium model suitable for Martian atmosphere are also considered [11, 12]. The code solves the fully Reynolds average Navier-Stokes (RANS) equations on hybrid grids by means of the finite volume approach; it uses a flux difference splitting (FDS) second-order upwind scheme for the spatial reconstruction of convective terms, while for the diffusive fluxes, a cell-centred scheme is applied.

In the present CFD analysis, an alternative way to compute the flux vector is considered by using a flux-vector splitting scheme, namely, Advection Upstream 
Splitting Method (AUSM). It provides an exact resolution of contact and shock discontinuities and it is less susceptible to Carbuncle phenomena. Implicit solver formulation is available and considered in the computations of this work. Indeed, due to broader stability characteristics of the implicit formulation, a converged steady-state solution can be obtained much faster using the implicit formulation rather than the explicit one. Global transport properties of the gas mixture rely on semiempirical rules such as Wilke's mixing rule for viscosity and thermal conductivity [5]. The viscosity and thermal conductivity of the $i$ th species is obtained by Kinetic Theory of gases [5]. For the diffusion coefficient of the $i$ th species in the mixture, the multicomponent diffusion coefficient is applied, where species mass diffusivity is evaluated by Kinetic Theory [5]. Flowfield chemical reactions proceed with forward rates that are expressed in the Arrhenius form and reaction rate parameters are due to Park et al. [13].

In particular, a number of in-house modifications (i. e., UDF) have been developed to account for both the thermal nonequilibrium in the flow and radiative equilibrium temperature at wall, since both vibrational nonequilibrium and wall radiative cooling boundary conditions are not basic code features. In the UDF, vibrational relaxation is modeled using a Landau-Teller formulation, where relaxation times are obtained from Millikan and White, assuming simple harmonic oscillators [5]; whereas to account for wall radiative cooling, during numerical simulations, the wall temperature is calculated by Stephan-Boltzman law and is implemented by means of a Newton-Raphson approach.

Finally, in order to assess numerical solution convergence, equation residuals and aerodynamic coefficients (i. e., $C_{L}, C_{D}$, and $C_{M}$ ) have been monitored during iterations. Solution convergence is assumed when equation residuals drop more than three orders of magnitude and the aerodynamic coefficients plots are flat for enough iterations.

\section{FLOWFIELD ANALYSIS}

The CFD analyses have been performed to assess the aerothermal environment that the MBS experiments during the descent, thus evaluating several surface loading distributions (e.g., pressure and heat flux). To this end, several fully three-dimensional numerical computations, both for perfect and chemically reacting gas approximations, have been performed.

\subsection{Freestream Conditions}

The flight scenario considered so far is summarized in Fig. 5. It refers to a vehicle entering the Mars atmosphere both from an elliptic orbit (EO), e.g., 

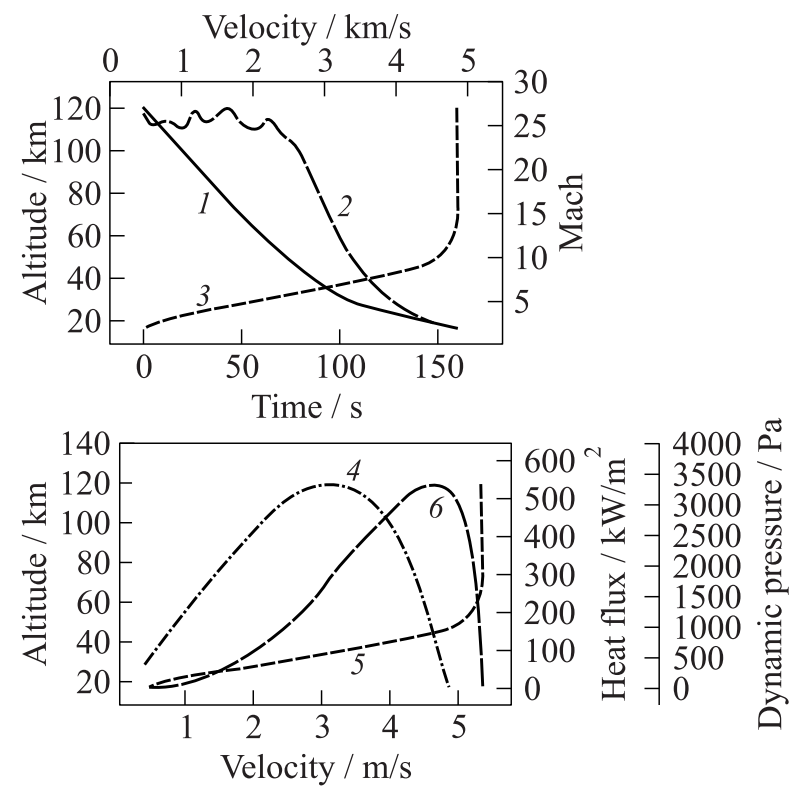

(a)

Velocity / km/s
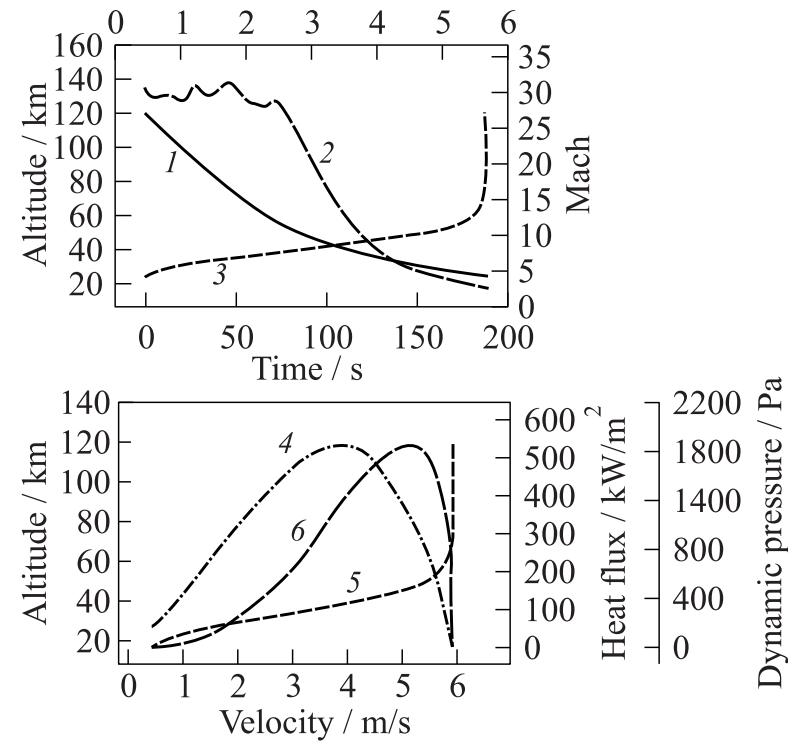

(b)

Figure 5 The MBS flight scenario: $(a)$ EO and (b) HO: 1 - altitude vs. time; 2 Mach vs. time; 3 - altitude vs. velocity; 4 - dynamic pressure; 5 - altitude; and 6 - heat flux 
Table 1 The CFD freestream conditions [14]

\begin{tabular}{|c|c|c|c|c|c|c|c|}
\hline $\begin{array}{c}\text { Simulation } \\
\text { ID }\end{array}$ & Gas model & Mach & AoA & $\begin{array}{l}\text { Altitude, } \\
\text { km }\end{array}$ & $\begin{array}{c}\text { Pressure, } \\
\mathrm{Pa}\end{array}$ & $\begin{array}{c}\text { Temperature, } \\
\text { K }\end{array}$ & $\begin{array}{c}\text { Density, } \\
\mathrm{g} / \mathrm{m}^{3}\end{array}$ \\
\hline 1 & Perfect gas & 10 & $10^{\circ}$ & 10 & 284.19 & 227.55 & 6.50 \\
\hline 2 & & 15 & $40^{\circ}$ & 60 & 3.16 & 116.55 & 0.141 \\
\hline 3 & & 20 & $10^{\circ}$ & 60 & 3.16 & 116.55 & 0.141 \\
\hline 4 & & 20 & $20^{\circ}$ & 60 & 3.16 & 116.55 & 0.141 \\
\hline 5 & & 20 & $30^{\circ}$ & 60 & 3.16 & 116.55 & 0.141 \\
\hline 6 & & 20 & $40^{\circ}$ & 60 & 3.16 & 116.55 & 0.141 \\
\hline 7 & & 22 & $40^{\circ}$ & 50 & 3.16 & 116.55 & 0.141 \\
\hline 8 & $\overline{\mathrm{EOPH}}$ & 22 & $40^{\circ}$ & 44.2 & 13.09 & 151.53 & 0.449 \\
\hline 9 & $\mathrm{HOPH}$ & 26 & $40^{\circ}$ & 52.1 & 6.43 & 134.00 & 0.250 \\
\hline$\overline{10}$ & Peacting gas & $\overline{10}$ & $10^{\circ}$ & $\overline{10}$ & 284.19 & 227.55 & $\overline{6.50}$ \\
\hline 11 & & 10 & $20^{\circ}$ & 10 & 284.19 & 227.55 & 6.50 \\
\hline 12 & & 10 & $30^{\circ}$ & 10 & 284.19 & 227.55 & 6.50 \\
\hline 13 & & 10 & $40^{\circ}$ & 10 & 284.19 & 227.55 & 6.50 \\
\hline 14 & & 20 & $10^{\circ}$ & 60 & 3.16 & 116.55 & 0.141 \\
\hline 15 & & 20 & $20^{\circ}$ & 60 & 3.16 & 116.55 & 0.141 \\
\hline 16 & & 20 & $30^{\circ}$ & 60 & 3.16 & 116.55 & 0.141 \\
\hline 17 & & 20 & $40^{\circ}$ & 60 & 3.16 & 116.55 & 0.141 \\
\hline 18 & $\overline{\mathrm{NCW}}$ & 22 & $40^{\circ}$ & 44.2 & 13.09 & 151.63 & 0.449 \\
\hline 19 & EOPH $\overline{\text { FCW }}$ & 22 & $40^{\circ}$ & 44.2 & 13.09 & 151.63 & 0.449 \\
\hline 20 & $\mathrm{NCW}$ & 26 & $40^{\circ}$ & 52.1 & 6.43 & 134.09 & 0.250 \\
\hline 21 & $\overline{\mathrm{FCW}}$ & 26 & $40^{\circ}$ & 52.1 & 6.43 & 134.09 & 0.250 \\
\hline
\end{tabular}

Remarks: EOPH - EO peak heating conditions; HOPH - HO peak heating conditions; $\mathrm{NCW}$ - noncatalytic wall: FCW - fully catalytic wall.

a planetary entry after aerobraking, and hyperbolic orbit $(\mathrm{HO})$, e.g., a direct planetary entry.

In particular, the freestream conditions considered in the present CFD analysis, according to both space-based and trajectory-based design approaches, are summarized in Table 1.

Therefore, 21 CFD numerical simulations (both Euler and Navier-Stokes) have been performed both in a trajectory-based design approach (i. e., simulation ID 1 to 7 and 10 to 17) and space-based design approach (i. e., simulation ID 8, 9 and 18 to 21) [7, 14]. In particular, the latter simulations refer to trajectory peak heating conditions both for EO and HO, needed to address the vehicle TPS design (not reported in this paper). Preliminary result of the vehicle aeroheating analysis can be found in $[15,16]$.

All CFD computations have been carried out considering radiative cooling at the vehicle wall (i.e., $\dot{q}_{\mathrm{cw}}=\sigma \varepsilon T_{w}^{4}$ ). 
For the reacting gas computations, the Martian atmosphere has been considered as a mixture of $95.7 \%$ Carbon dioxide, $1.6 \%$ Argon, and 2.7\% Nitrogen. The flow has been modeled as a reacting gas mixture of 9 species $\left(\mathrm{Ar}, \mathrm{CO}_{2}, \mathrm{~N}_{2}\right.$, $\mathrm{O}_{2}, \mathrm{CO}, \mathrm{NO}, \mathrm{N}, \mathrm{O}$, and $\mathrm{C}$ ). Reaction mechanism and chemical kinetics taken into account in the present work are from $[6,13]$.

Nonequilibrium computations have been performed since one of the most challenging problem facing the design of atmospheric entry vehicle is the phenomenon of "real gas behavior." At hypersonic speeds, the shock wave produced ahead of the vehicle suddenly elevates the gas temperature in the shock layer.

So, the gas thermal energy may be comparable with the energy associated with a whole range of gas chemical processes such as: molecular vibrational excitation; dissociation of atmospheric molecules into their atomic forms; formation of other chemical species through recombination reactions; and ionization of both molecular and atomic species [17]. Therefore, the gas mixture has to be considered in thermal and chemical nonequilibrium.

Finally, CFD analysis of the MBS was preceded by a code validation phase performed considering the available numerical and experimental data for the Mars Pathfinder probe at entry peak heating conditions, as summarized in [1820].

\subsection{Numerical Results}

The aerodynamic analysis of MBS is shown in terms of lift $\left(C_{L}\right), \operatorname{drag}\left(C_{D}\right)$, and pitching moment $\left(C_{M y}\right)$ coefficients which are calculated as follows:

$$
\begin{aligned}
C_{i} & =\frac{F_{i}}{(1 / 2) \rho_{\infty} v_{\infty}^{2} S_{\mathrm{ref}}}, & i=L, D ; \\
C_{M j} & =\frac{M_{j}}{(1 / 2) \rho_{\infty} v_{\infty}^{2} L_{\mathrm{ref}} S_{\mathrm{ref}}}, & j=Y .
\end{aligned}
$$

The reference parameters $L_{\text {ref }}$ (e.g., longitudinal reference length) and $S_{\text {ref }}$ (e.g., reference surface) are the vehicle length (i. e., $8 \mathrm{~m}$ for both configurations) and planform area (i. e., $31.7 \mathrm{~m}^{2}$ for LB-A and $32.0 \mathrm{~m}^{2}$ for LB-B). The pitching moment is computed from the vehicle nose (i. e., 0,0,0) and also considering the vehicle center of gravity $(\mathrm{CoG})$ at different percentage of vehicle length in order to address a CoG sensitivity analysis as well.

The MBS aerodynamic results, provided by engineering-based analysis, cover $\alpha$ ranging from $0^{\circ}$ to $50^{\circ}$.

On the other hand, present CFD computations for the MBS have been carried out on a 3D multiblock (up to 62 blocks) structured grids similar to those shown in Fig. 6. In particular, Fig. 6 shows a closeup view of the $3 \mathrm{D}$ mesh on the vehicle surface and pitch plane (LB-B only). 

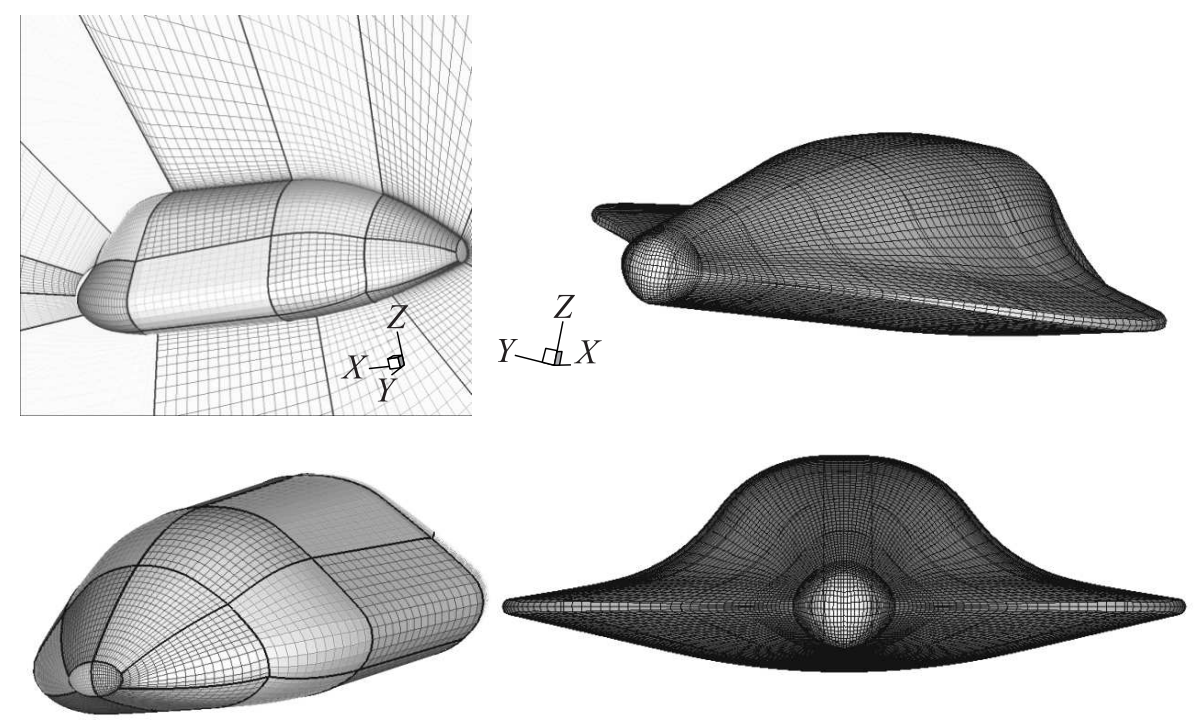

Figure 6 The computational mesh domains for Euler CFD simulations

All the mesh domains have been generated with the commercial software package ANSYS ICEM-CFD ${ }^{\circledR}$ and are characterized by an overall number of about $1.2 \cdot 10^{6}$ cells (half body).

Both computational domains are tailored for the free-stream conditions of Table 1. The distribution of surface grid points has been dictated by the level of resolution desired in various areas of the vehicle such as the stagnation region and the base fillet one, according to the computational scopes. Grid refinement in strong gradient regions of flowfield has been made through a solution adaptive approach.

Preliminary results of CFD simulations performed so far are summarized hereinafter. For example, Fig. 7 shows CFD (perfect gas) results for $\mathrm{M}_{\infty}=22$ and $\alpha=40^{\circ}$ concerning the configuration LB-A. Figure $7 a$ reports pressure coefficient contours $\left(C_{p}\right)$ on the vehicle surface and on two cross sections; whereas in Fig. $7 b, C_{p}$ contours on the vehicle surface and Mach number contours on three cross sections have been shown.

Looking at the contours field of Fig. 7, the shock layer that takes place past the vehicle at $\mathrm{M}_{\infty}=22$ and $\alpha=40^{\circ}$ can be clearly inferred, as in the case of both Figs. 8 and 9. In these figures, Mach number contours are shown on a vehicle cross section at $x=3.28 \mathrm{~m}$ (from nose) and on a section at $z=-0.1 \mathrm{~m}$ in order to identify the MBS bow shock also in the vehicle planform plane. Note that those sections also allow assessing the cause of the overpressure that takes place on the vehicle windside highlighted in Fig. 7. 

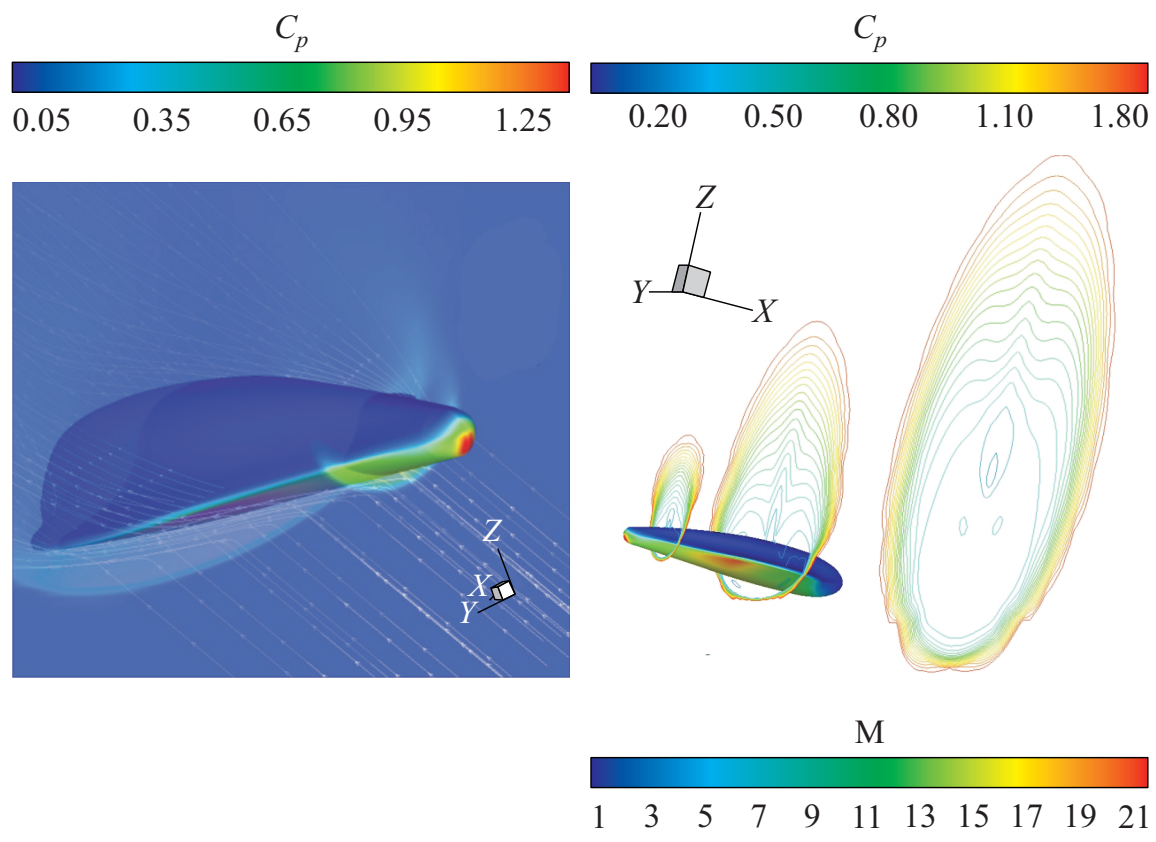

(a)
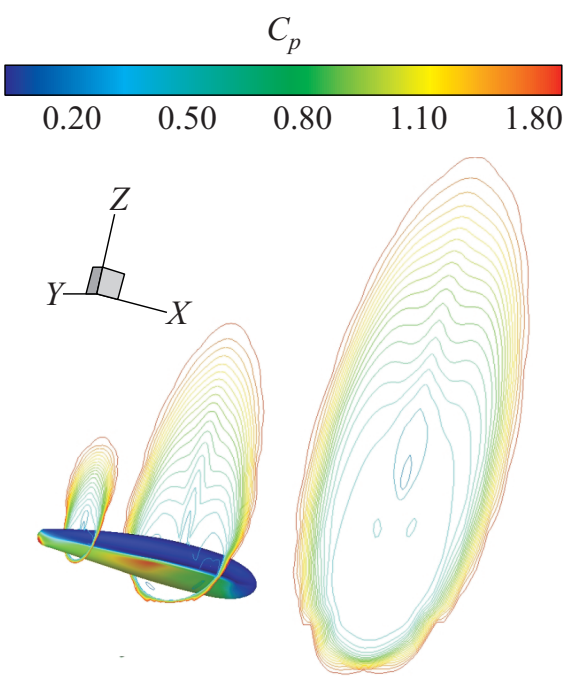

(b)

Figure 7 Pressure coefficient contours $\left(C_{p}\right)$ on LB-A surface and on two cross sections $(a)$ and $C_{p}$ contours on vehicle surface and Mach number contours on three cross sections $(b)$ at $\mathrm{M}_{\infty}=22$ and $\alpha=40^{\circ}$.

Indeed, results reported in Figs. 8 and 9 suggest that those pressure overshoots depend on the belly side shape and not on a shock-shock interaction phenomenon because of the shock shape reported in the vehicle planform plane does not impinge on the vehicle shape.

Figure 10 shows the static temperature contours on the vehicle (LB-A) symmetry plane and static pressure contours on the vehicle surface at $\mathrm{M}_{\infty}=20$ and $\alpha=20^{\circ}$, considering the Mars atmosphere as a reacting gas mixture. As shown, streamtraces are reported to point out a flowfield structure past the vehicle at those flight conditions.

At the same freestream conditions, Fig. 11 reports the contours of $\mathrm{N}_{2}, \mathrm{CO}_{2}$, $\mathrm{O}$, and $\mathrm{CO}$ mass fractions on the pitch plane of LB-A concept, thus accounting for chemical dissociation of the flow in the shock layer. As a consequence, flow dissociation determines a large density ratio $\varepsilon$ across the bow shock com- 

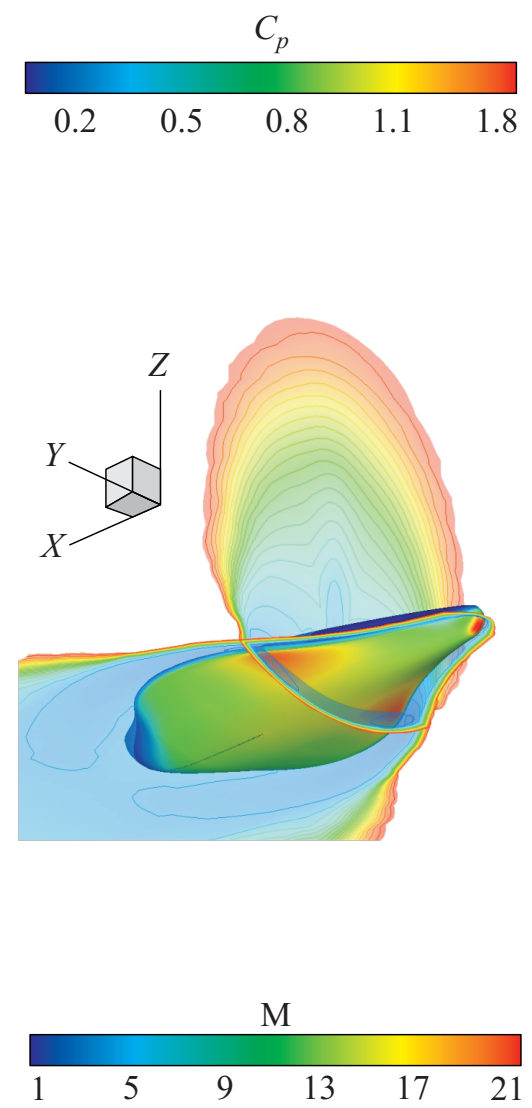

Figure 8 Pressure coefficient contours $\left(C_{p}\right)$ on LB-A surface and Mach number contours on two flowfield sections at $\mathrm{M}_{\infty}=22$ and $\alpha=40^{\circ}$. Windside view.

pared with a flow of the same gas where no dissociation takes place, resulting in a thinner shock layer around the entry vehicle (e.g., lower standoff distance).

Under conditions where the dissociation exists, the aerodynamics of vehicle depends primarily on shock density ratio. In fact, the change of aerodynamic characteristics is the result of the change in the surface pressure acting on the vehicle forebody [5]. 


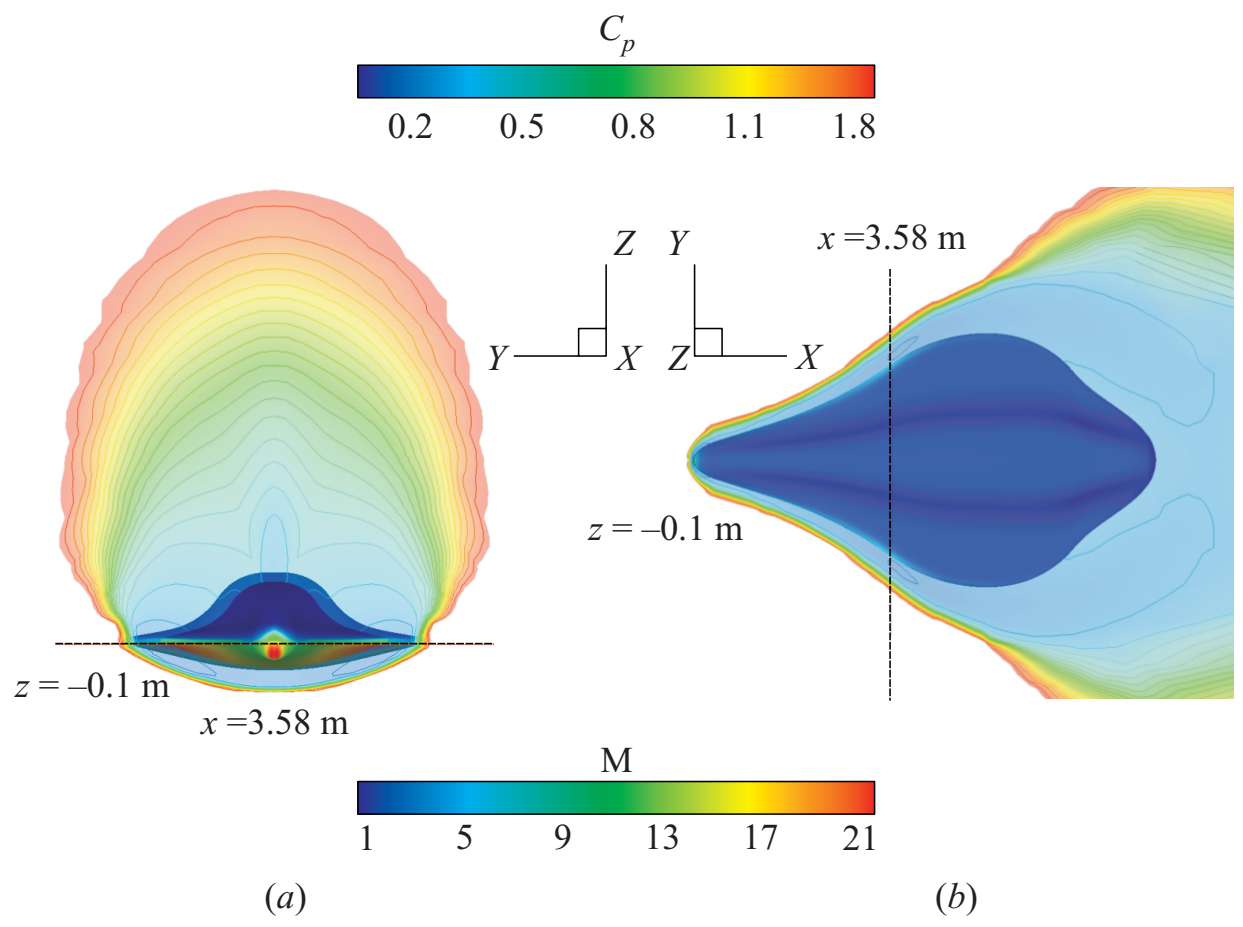

Figure 9 Pressure coefficient contours $\left(C_{p}\right)$ on LB-A surface and Mach number contours on two flowfield sections. Front $(a)$ and up $(b)$ views; $\mathrm{M}_{\infty}=22$ and $\alpha=40^{\circ}$.

The curves of lift and drag coefficients are shown in Fig. 12 for $\mathrm{M}_{\infty}=20$ and 60-kilometer altitude. These curves collect MBS (LB-A) aerodynamic coefficients compared with available numerical data both for perfect gas and reacting gas approximations, reported in order to highlight the accuracy of both numerical and engineering-based results [16]. As one can see, engineering and numerical data agree very well, thus confirming that engineering-based estimations represent reliable preliminary aerodynamics of a Mars entry vehicle. Moreover, the results of the comparison highlight also that high enthalpy effects only slightly increase the aerodynamic lift and drag coefficients at those flight conditions due to its high streamlined aeroshape.

As far as CFD results for the second configuration (i. e., LB-B) are concerned, Fig. 13 shows the Mach number contour field that takes place around the vehicle 


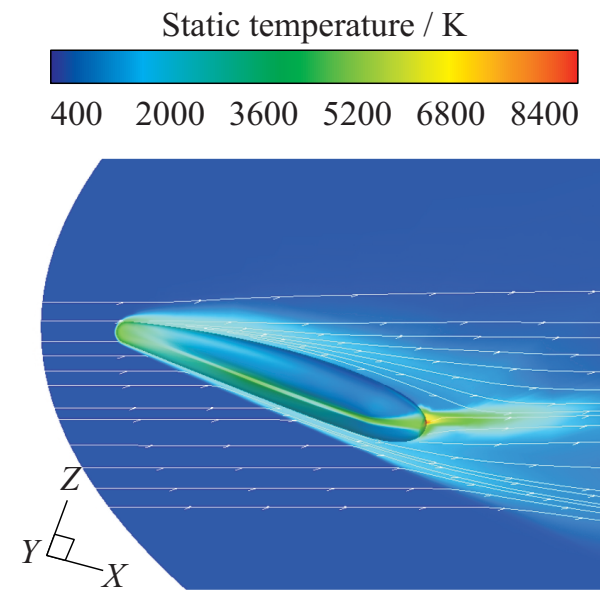

(a)
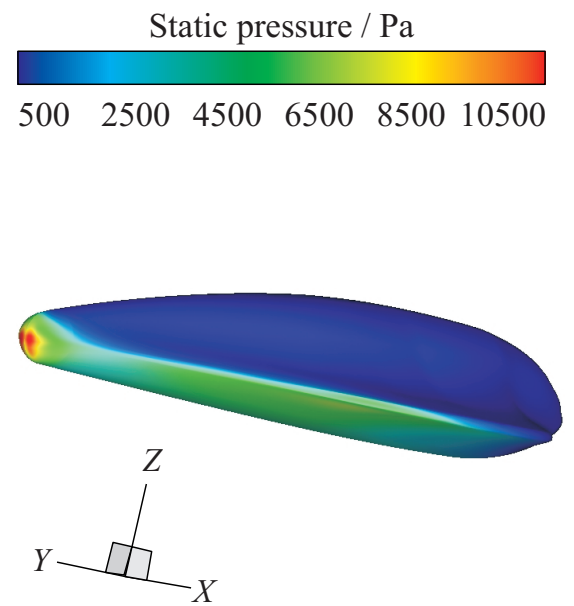

(b)

Figure 10 Static temperature field on vehicle (LB-A) symmetry plane (a) and static pressure contour on vehicle surface for nonequilibrium reacting gas (b) at $\mathrm{M}_{\infty}=20$ and $\alpha=20^{\circ}$.

when it is flying at the peak heating conditions of the entry by EO (e.g., $\mathrm{M}_{\infty}$ $=22, \alpha=40^{\circ}$, and $H=44.20 \mathrm{~km}$ ). In particular, Fig. $13 a$ shows the Mach contour field on the vehicle pitch plane while Fig. $13 b$ gives an idea of the bow shock shape that envelopes the vehicle, since the Mach field is reported on three different flowfield cross sections.

As shown, a thin shock layer envelopes the entry vehicle with a strong expansion that characterizes the windside flow at the end of the vehicle concept.

The CO mass fraction field around the vehicle for the same freestream conditions is shown in Fig. 14 where some streamtraces colored by Mach number are also reported.

As one can see, the $\mathrm{CO}$ concentration reaches its maximum value close to the body and evolves through the vehicle wake flow [21].

Figure 15 shows the temperature comparison among nonequilibrium flow (right side of pilot) and perfect gas computation, evaluated at three flowfield cross sections $(x=1.5,5.5$, and $9.5 \mathrm{~m})$. It is clearly evident how real gas phenomena affect the vehicle shock layer, as discussed above.

Curves of lift, drag, and pitching moment coefficients are shown in Fig. 16. As shown, high enthalpy effects slightly increase aerodynamic lift, drag, and pitching moment coefficients. Vehicle aerodynamics is summarized in Table 2. 


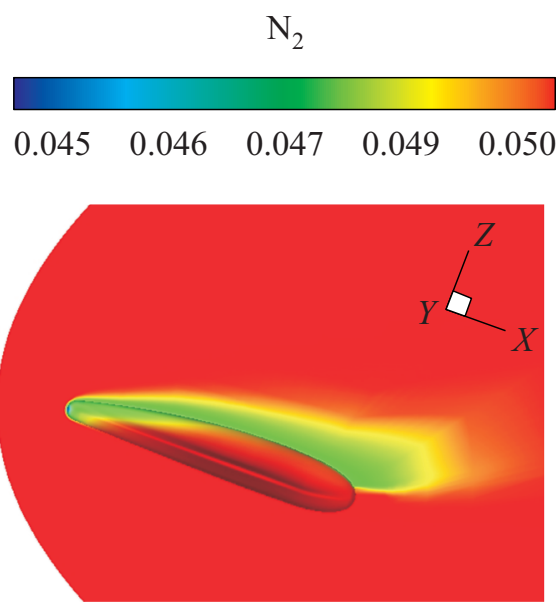

(a)
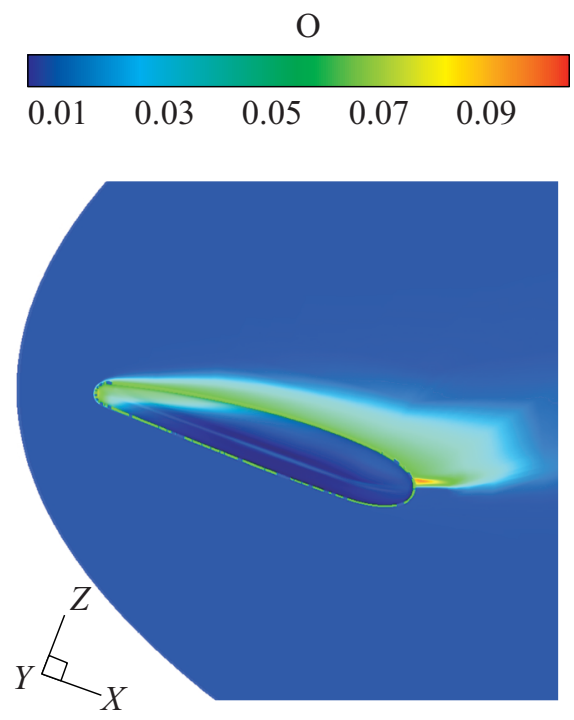

(c)

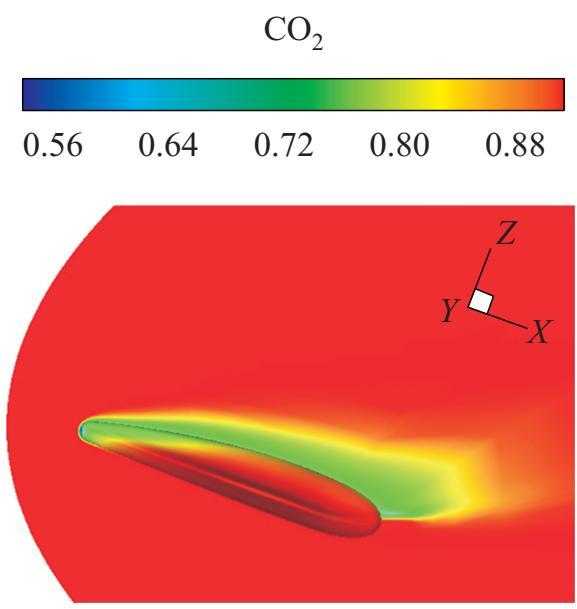

(b)
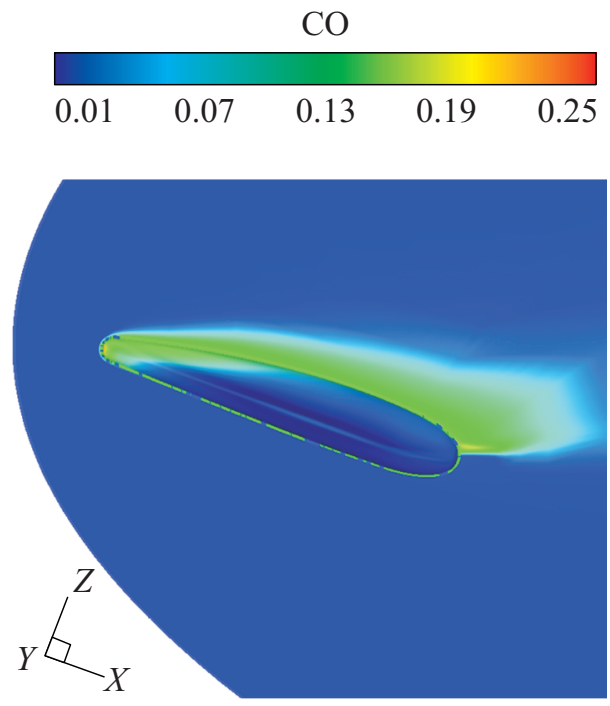

(d)

Figure 11 Contours of $\mathrm{N}_{2}(a), \mathrm{CO}_{2}(b), \mathrm{O}(c)$, and $\mathrm{CO}(d)$ mass fractions on MBS (LB-A) pitch plane at $\mathrm{M}_{\infty}=20$ and $\alpha=20^{\circ}$. 


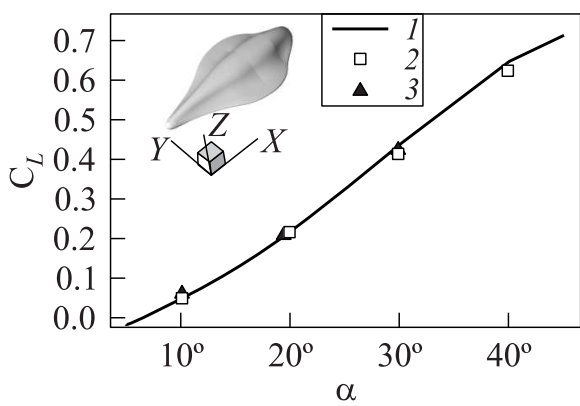

(a)

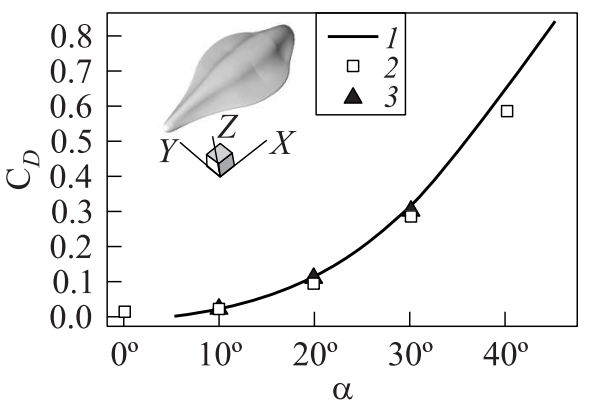

(b)

Figure 12 Comparison between HPM (1) and CFD results for perfect (2) and reacting (3) gas approximations at $\mathrm{M}_{\infty}=20$ and $H_{\infty}=60 \mathrm{~km}$

$\mathrm{M}$

$\begin{array}{llllllllllllllllllll}1 & 2 & 3 & 4 & 6 & 6 & 8 & 9 & 10 & 11 & 12 & 13 & 14 & 15 & 16 & 17 & 18 & 19 & 20 & 21\end{array}$

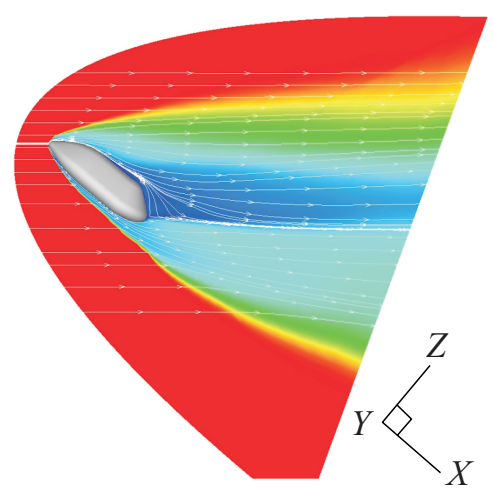

(a)

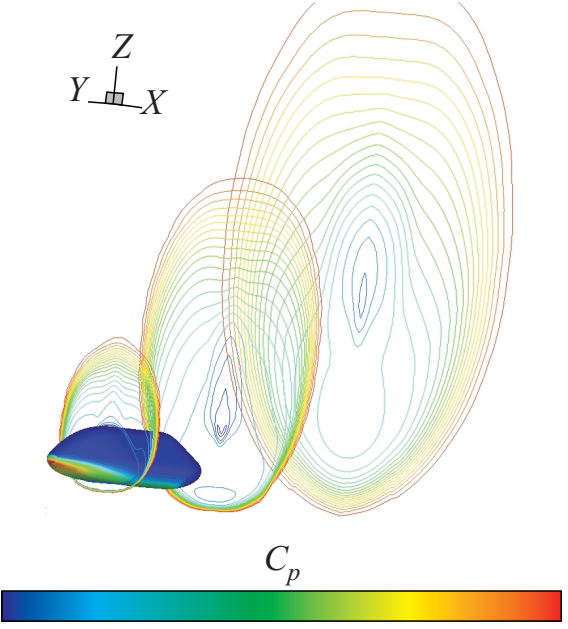

$\begin{array}{llllllllll}0.1 & 0.3 & 0.5 & 0.7 & 0.9 & 1.1 & 1.3 & 1.5 & 1.7\end{array}$

(b)

Figure 13 Mach contours on the vehicle pitch plane $(a)$ and three flowfield cross sections $(b)$ at the EOPH conditions $\left(\mathrm{M}=22, \alpha=40^{\circ}\right.$, and $\left.H=44.2 \mathrm{~km}\right)$. 


\section{$\mathrm{CO}$}

\section{$\begin{array}{lllllllllll}0.01 & 0.04 & 0.07 & 0.10 & 0.13 & 0.16 & 0.19 & 0.22 & 0.25 & 0.28 & 0.31\end{array}$}

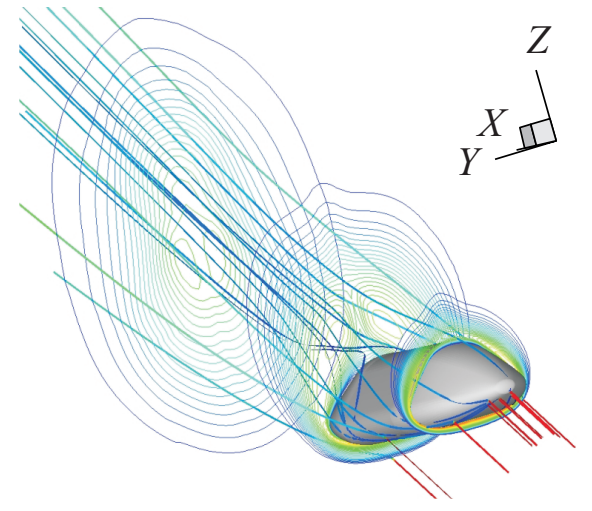

M

$\begin{array}{llllllllllllllllllll}12 & 3 & 5 & 6 & 7 & 8 & 9 & 10 & 11 & 12 & 13 & 14 & 15 & 16 & 17 & 18 & 19 & 20 & 21\end{array}$

Figure 14 CO mass fraction at the EOPH conditions on three cross sections with streamtraces coloured by Mach number.

As far as vehicle aerodynamic performance comparisons are concerned, Figs. 17 and 18 show the pitching moment coefficient and the aerodynamic efficiency for both MBS concepts.

Results reported in Fig. $17 a$ highlight that the concept LB-A is statically stable in longitudinal flight (e.g., $C_{M_{\alpha}}<0$ ) for $\alpha \geq 20^{\circ}$ and CoG located up to half of the vehicle length; whereas if the CoG is at $55 \%$ of $L_{\text {ref }}$ (maximum allowable $\mathrm{CoG}$ excursion), the AoAs must be higher than $30^{\circ}$ to guarantee static stability flight conditions. In particular, the AoA at which the moment coefficient about the $\mathrm{CoG}$ is equalled to zero (i. e., trim condition) are appreciatively $20^{\circ}$ and $40^{\circ}$ for CoG at $50 \% L_{\text {ref }}$ and $55 \% L_{\text {ref }}$, respectively.

On the other hand, Fig. $17 b$ shows that at hypersonic speed, the LB-B concept is statically stable in longitudinal flight for $\alpha \geq 20^{\circ}$ and CoG located up to $40 \%$ of vehicle length, whereas if the CoG is at $50 \%$ of $L_{\text {ref }}$ (maximum allowable CoG excursion), the AoAs must be higher than $40^{\circ}$. In particular, trim AoAs are appreciatively $20^{\circ}$ and $40^{\circ}$ for CoG at $40 \% L_{\text {ref }}$ and $45 \% L_{\text {ref }}$, respectively. 


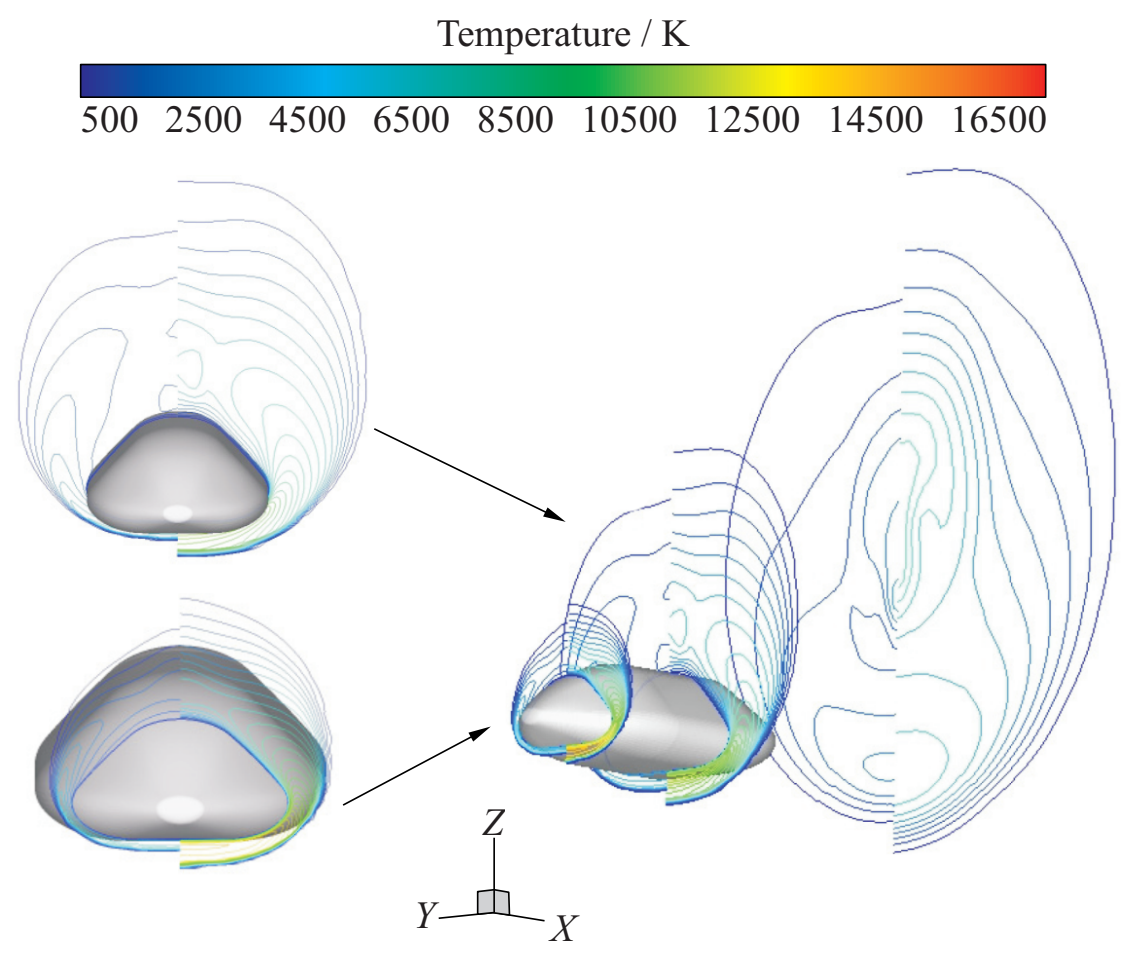

Figure 15 Temperature comparison between nonequilibrium flow (right side of pilot) and perfect gas computations at $x=1.5,5.5$, and $9.5 \mathrm{~m}$ flowfield cross sections.

Finally, Fig. 18 shows the lift-to-drag ratio $(L / D)$ comparison between LB-A and LB-B vehicle concepts. As one can see, the former MBS features a better aerodynamics performance at low AoA (say, $10^{\circ} \leq \alpha \leq 35^{\circ}$ ) as expected due to its more streamlined configuration and different aeroshape.

\section{CONCLUDING REMARKS}

The paper deals with the flowfield analysis of two braking systems for manned exploration missions to Mars. A number of fully 3D Navier-Stokes and Euler CFD computations of the hypersonic flowfield past two LB vehicles have been performed for several freestream conditions of a proposed Mars entry loading environment. These evaluations have been aimed at carrying out only a preliminary design of the MBS configuration, in compliance with the Phase-A design level. The range between Mach 10 and Mach 26 has been analyzed to provide aero- 


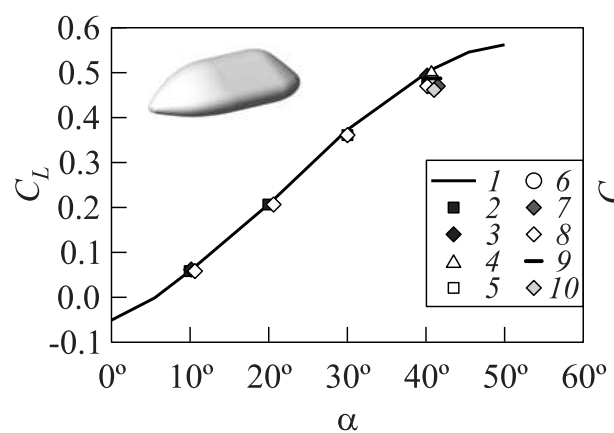

(a)

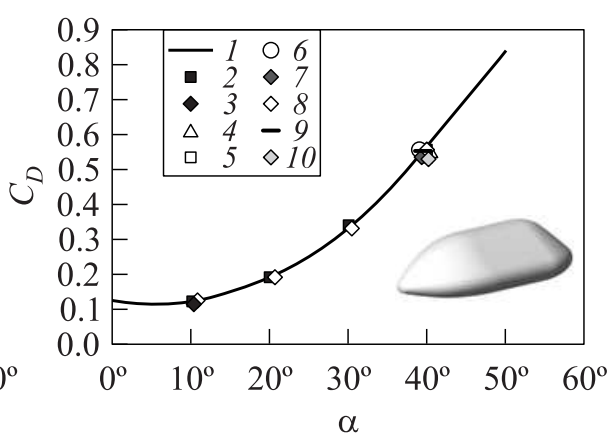

(b)

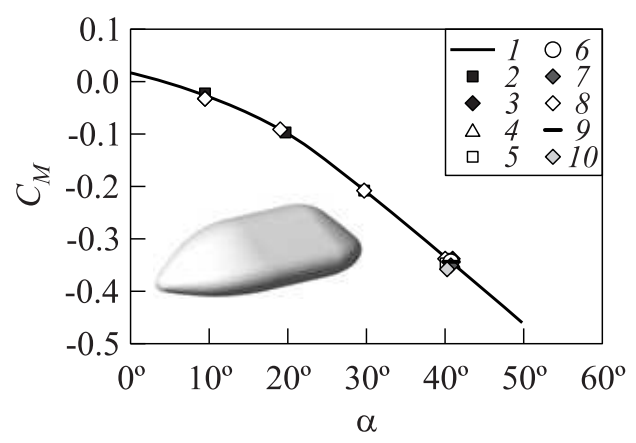

(c)

Figure 16 Lift $(a)$, drag $(b)$, and pitching moment $(c)$ coefficients vs. $\alpha$. Comparison between HPM (1) and CFD results for perfect $(2-$ Euler $\mathrm{M}=9 ; 3-10 ; 4-15$; $5-\mathrm{M}=22, H=60 \mathrm{~km} ; 6-\mathrm{M}=22, \mathrm{EOPH}$; and $7-\mathrm{M}=26, \mathrm{HOPH})$ and nonequilibrium gas computations $(8-\mathrm{M}=10 ; 9-\mathrm{M}=22$, EOPH; and $10-$ $\mathrm{M}=26, \mathrm{HOPH})$

dynamic performance according to both the space-based and trajectory-based design approaches.

The results reported in the paper are useful for providing numerical data for further understanding the fluid dynamics process, as those of high enthalpy and vehicle shape effects, involved in the design analysis of LB vehicles for the exploration of Mars.

\section{REFERENCES}

1. Polishchuka, G., K. Pichkhadzea, V. Vorontsovb, and K. Pavela. 2006. Proposal on application of Russian technical facilities for International Mars Research Program for 2009-2015. Acta Astronautica 59:113-18. 
Table 2 Vehicle aerodynamics

\begin{tabular}{|c|c|c|c|c|c|c|c|}
\hline$\overline{\text { Cons }}$ & tions & Mach & $\overline{\mathrm{AoA}}$ & Altitude, $\mathrm{km}$ & $\overline{C_{L}}$ & $\overline{C_{D}}$ & $C_{M}{ }^{*}$ \\
\hline Perfect & & 10 & $10^{\circ}$ & 10.0 & 0.06150 & 0.13060 & -0.03170 \\
\hline & & 15 & $40^{\circ}$ & 60.0 & 0.47830 & 9.55360 & -0.32940 \\
\hline & & 22 & $40^{\circ}$ & 60.0 & 0.47930 & 0.55540 & -0.33010 \\
\hline & EOPH & 22 & $40^{\circ}$ & 44.2 & 0.48300 & 0.55500 & -0.33200 \\
\hline & HOPH & 26 & $40^{\circ}$ & 52.1 & 0.49920 & 0.55930 & -0.34330 \\
\hline Reactin & gas & $\overline{10}$ & $10^{\circ}$ & 10.0 & 0.05710 & 0.12450 & -0.02950 \\
\hline & & 10 & $20^{\circ}$ & 10.0 & 0.19940 & 0.18730 & -0.09930 \\
\hline & & 10 & $30^{\circ}$ & 10.0 & 0.35970 & 0.33420 & -0.20650 \\
\hline & & 10 & $40^{\circ}$ & 10.0 & 0.49340 & 0.56100 & -0.33770 \\
\hline EOPH & $\overline{\mathrm{NCW}}$ & $\overline{22}$ & $40^{\circ}$ & 44.2 & 0.48368 & 0.55014 & -0.32980 \\
\hline & $\mathrm{FCW}$ & 22 & $40^{\circ}$ & 44.2 & 0.48369 & 0.55018 & -0.32980 \\
\hline HOPH & $\overline{\mathrm{NCW}}$ & 26 & $40^{\circ}$ & 52.1 & 0.47122 & 0.53627 & -0.32220 \\
\hline & FCW & 26 & $40^{\circ}$ & 52.1 & 0.47875 & 0.54536 & -0.32560 \\
\hline
\end{tabular}

${ }^{*}$ Pole at vehicle nose.

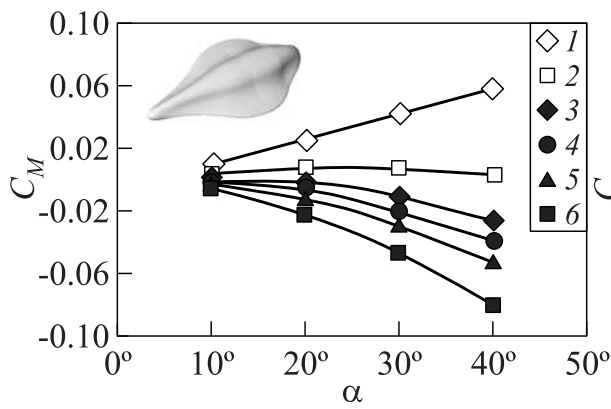

(a)

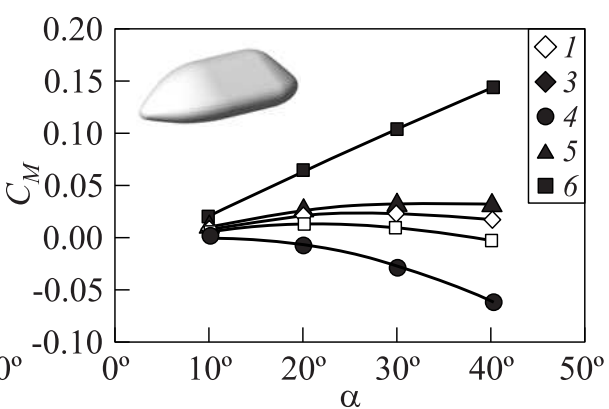

(b)

Figure 17 Pitching moment coefficients $C_{M}$ vs. $\alpha$ for LB-A $(a)$ and LB-B (b) concepts for CoG position at different percentage of vehicle length and at hypersonic flight conditions: $1-0.65 L_{\mathrm{ref}} ; 2-0.55 L_{\mathrm{ref}} ; 3-0.50 L_{\mathrm{ref}} ; 4-0.48 L_{\mathrm{ref}} ; 5-0.45 L_{\mathrm{ref}}$; and $6-0.40 L_{\mathrm{ref}}$

2. Viviani, A., and G. Pezzella. 2009. Aerodynamic analysis of a capsule vehicle for a manned exploration mission to Mars. AIAA Paper No. 2009-7386.

3. Viviani, A., L. Iuspa, G. Lanzillo, and G. Pezzella. 2011. Preliminary design of minimum weight configurations for a re-entry vehicle. Conference (International) of the European Aerospace Societies. Venice, Italy. CEAS 2011.

4. Hanley, G. M., and F. J. Lyon. 1964. The feasibility of spacecraft deceleration by aerodynamic braking at the planet Mars. AIAA Paper No. 64-479. 


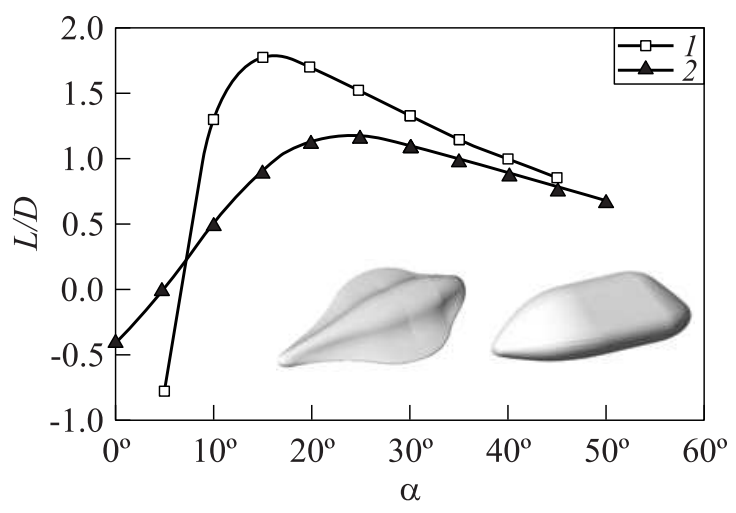

Figure 18 Lift-to-drag ratio vs. $\alpha(\mathrm{M}=20)$. Comparison between LB-A (1) and LB-B (2) concepts

5. Anderson, J. D. 1989. Hypersonic and high temperature gas dynamics. New York: McGraw-Hill Book Co.

6. Pezzella, G., and A. Viviani. 2011. Aerodynamic analysis of a Mars exploration manned capsule. Acta Astronautica 69:975-86.

7. Prabhu, D.K. 2004. System design constraints-trajectory aerothermal environments. RTO AVT/VKI Lecture Series in Critical Technologies for Hypersonic Vehicle Development.

8. Pezzella, G. 2011. Aerodynamic and aerothermodynamic trade-off analysis of a small hypersonic flying test bed. Acta Astronautica 69(3-4):209-22.

9. Bertin, J. J. 1994. Hypersonic aerothermodynamics. AIAA education ser.

10. FUENT 12.0 Theory Guide.

11. Mack, A. 2008. CFD Validation for $\mathrm{CO}_{2}$ re-entry applications. 2nd International ARA Days. Arcachon, France. AA-3-2008-37.

12. Hannemann, V., and A. Mack. 2009. Chemical nonequilibrium model of the Martian atmosphere. 6th European Symposium on Aerothermodynamics for Space Vehicles Proceedings. Versailles, France. ESA SP-659.

13. Park, C., J. T. Howe, R. L. Jaffe, and G. V. Candler. 1994. Review of chemicalkinetic problems of future NASA missions. II: Mars entries. J. Thermophys. Heat Transfer 8(1):9-23.

14. NASA-JPL. 1994. Mars Exploration Program. www.mars.jpl.nasa.gov.

15. Viviani, A., G. Pezzella, and C. Golia. 2010. Aerodynamic and thermal design of a space vehicle entering the Mars atmosphere. 5th Conference (International) on Thermal Engineering Theory and Applications. Marrakesh. Morocco. 
16. Viviani, A., G. Pezzella, and C. Golia. 2010. Aerothermodynamic analysis of a space vehicle for manned exploration missions to Mars. 27th Congress (International) of the Aeronautical Sciences, ICAS 2010. Nice, France.

17. Kustova, E. V., E. A. Nagnibeda, Y. D. Shevelev, and N. G. Syzranova. 2009. Comparison of non-equilibrium supersonic $\mathrm{CO}_{2}$ flows with real gas effects near a blunt body. 6th European Symposium on Aerothermodynamics for Space Vehicles. Versailles, France. ESA SP-659.

18. Gupta, R., K. Lee, and C. Scott. 1996. Aerothermal study of Mars pathfinder aeroshell. J. Spacecraft Rockets 33(1).

19. Gnoffo, P., K. Weilmuenster, R. Braun, and C. Cruz. 1996. Influence of sonicline location on Mars pathfinder probe aerothermodynamics. J. Spacecraft Rockets $33(2)$.

20. Gnoffo, P., R. Braun, K. Weilmuenster, R. Mitcheltree, W. Engelung, and R. Powell. 1998. Prediction and validation of Mars pathfinder hypersonic aerodynamic data base. 7th AIAA/ASME Joint Thermophysics and Heat Transfer Conference. Albuquerque, NM, USA.

21. Mitcheltree, R. A., and P. A. Gnoffo. 1995. Wake flow about the Mars pathfinder entry vehicle. J. Spacecraft Rockets $32(5)$. 